\title{
Correlation Analysis of Trp-cage Dynamics in Folded and Unfolded States
}

\section{Supporting Information}

Supplementary Figures and Table

Luigi L. Palese*

SMBNOS, University of Bari “Aldo Moro”, Piazza G. Cesare - Policlinico, 70124 Bari, Italy

\author{
AUTHOR INFORMATION \\ Corresponding Author: \\ *Luigi L. Palese: Email: luigileonardo.palese@uniba.it
}




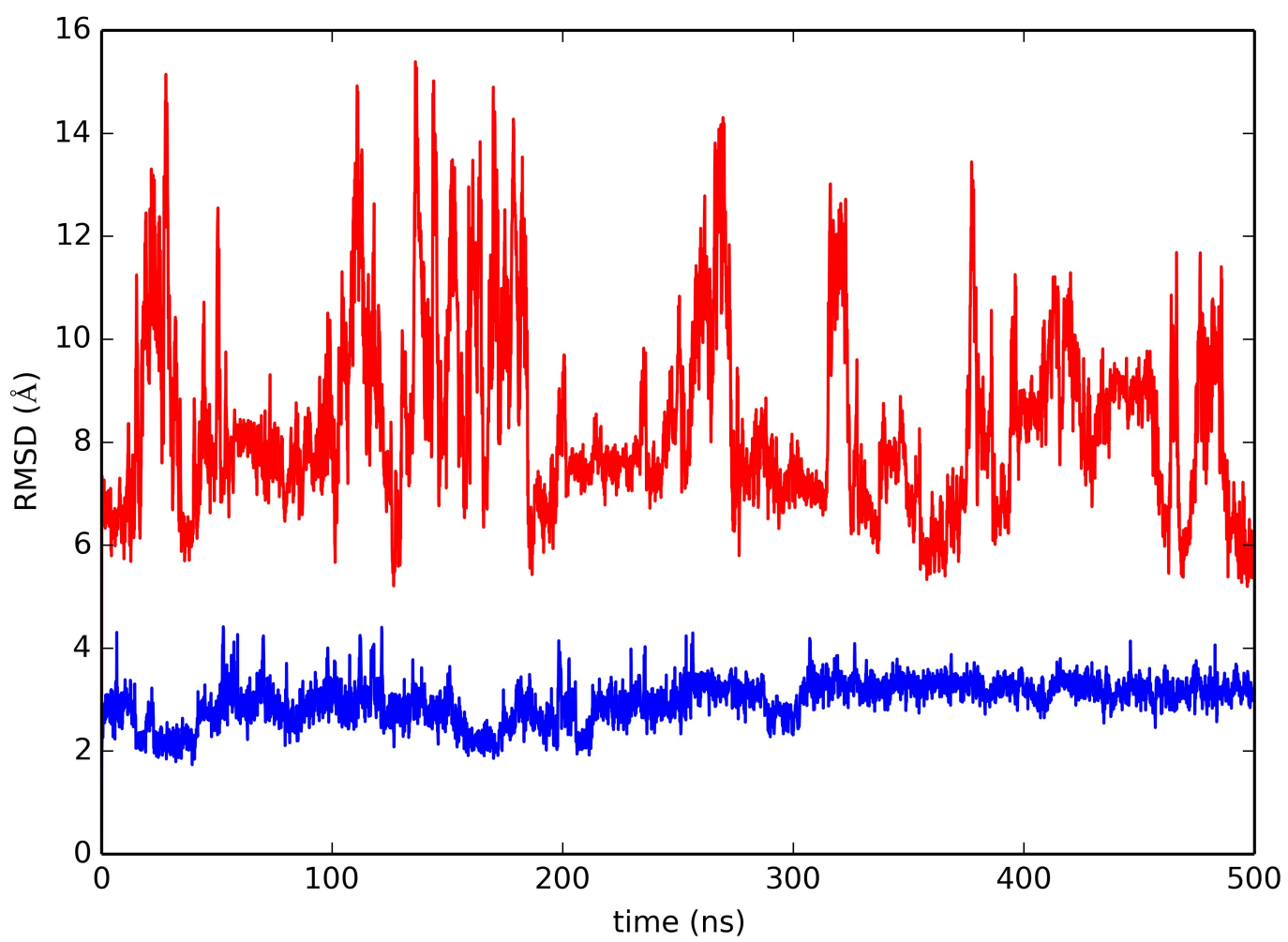

Figure S1. RMSD of the $300 \mathrm{~K}$ (blue line) and $335 \mathrm{~K}$ (red line) simulations discussed in the main text. 


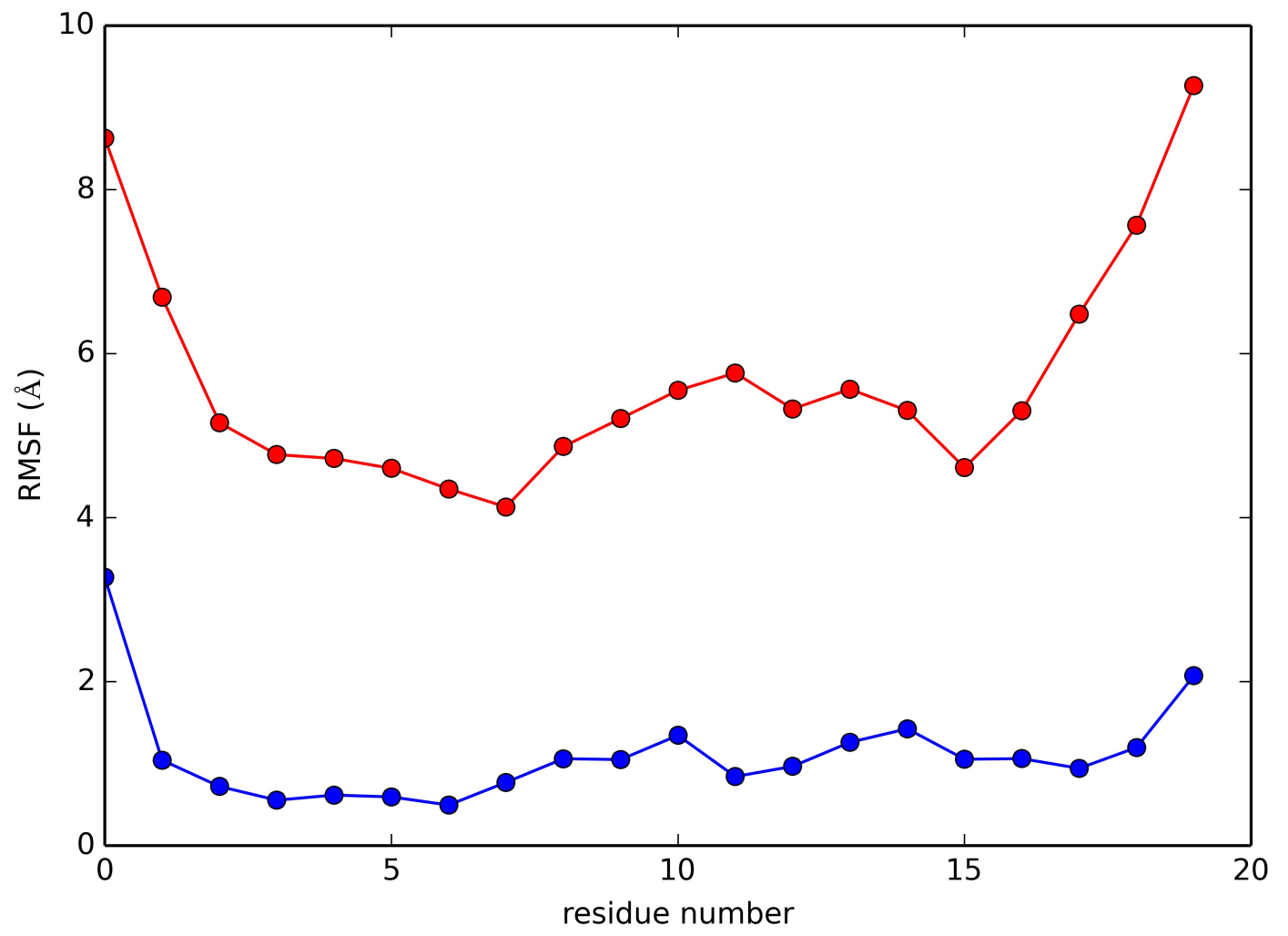

Figure S2. RMSF of the $300 \mathrm{~K}$ (blue line) and $335 \mathrm{~K}$ (red line) simulations discussed in the main text. 


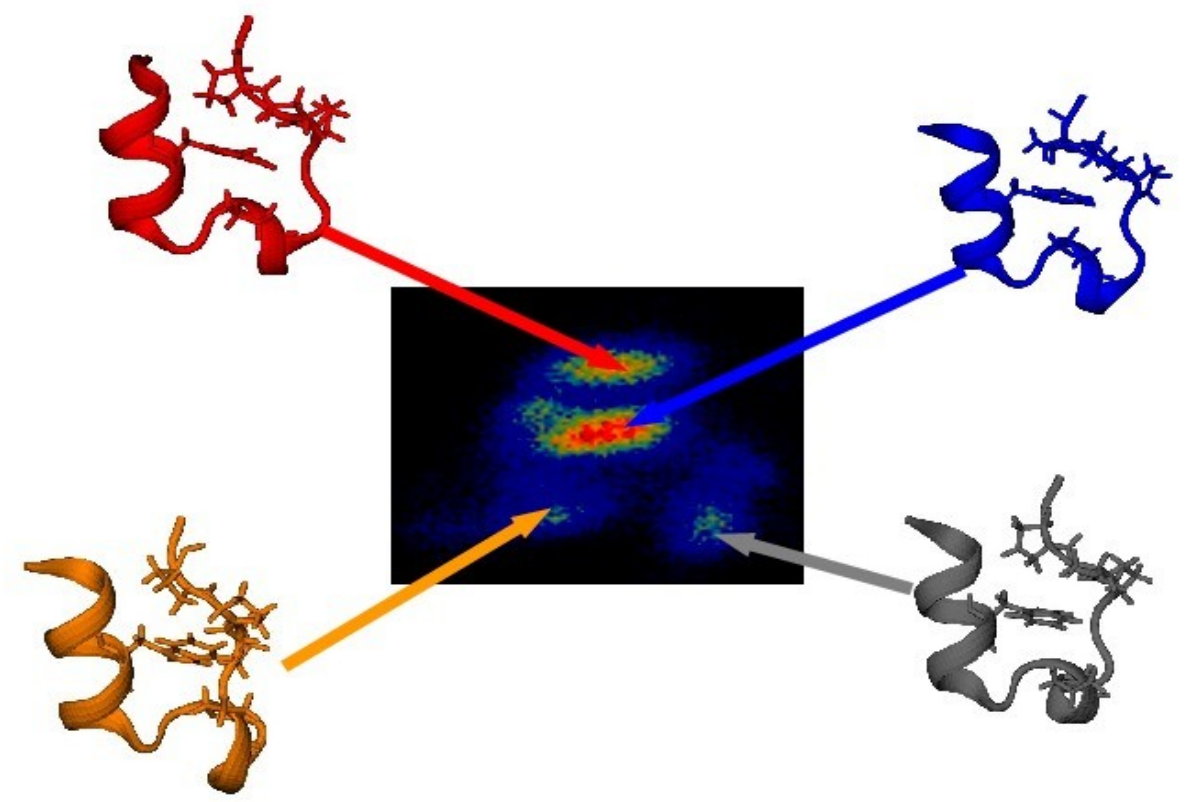

Figure S3. Representative structures of the major clusters visited in the simulation performed at $300 \mathrm{~K}$. Tryptophan and proline residues are plotted in stick representation. See text for further details. 


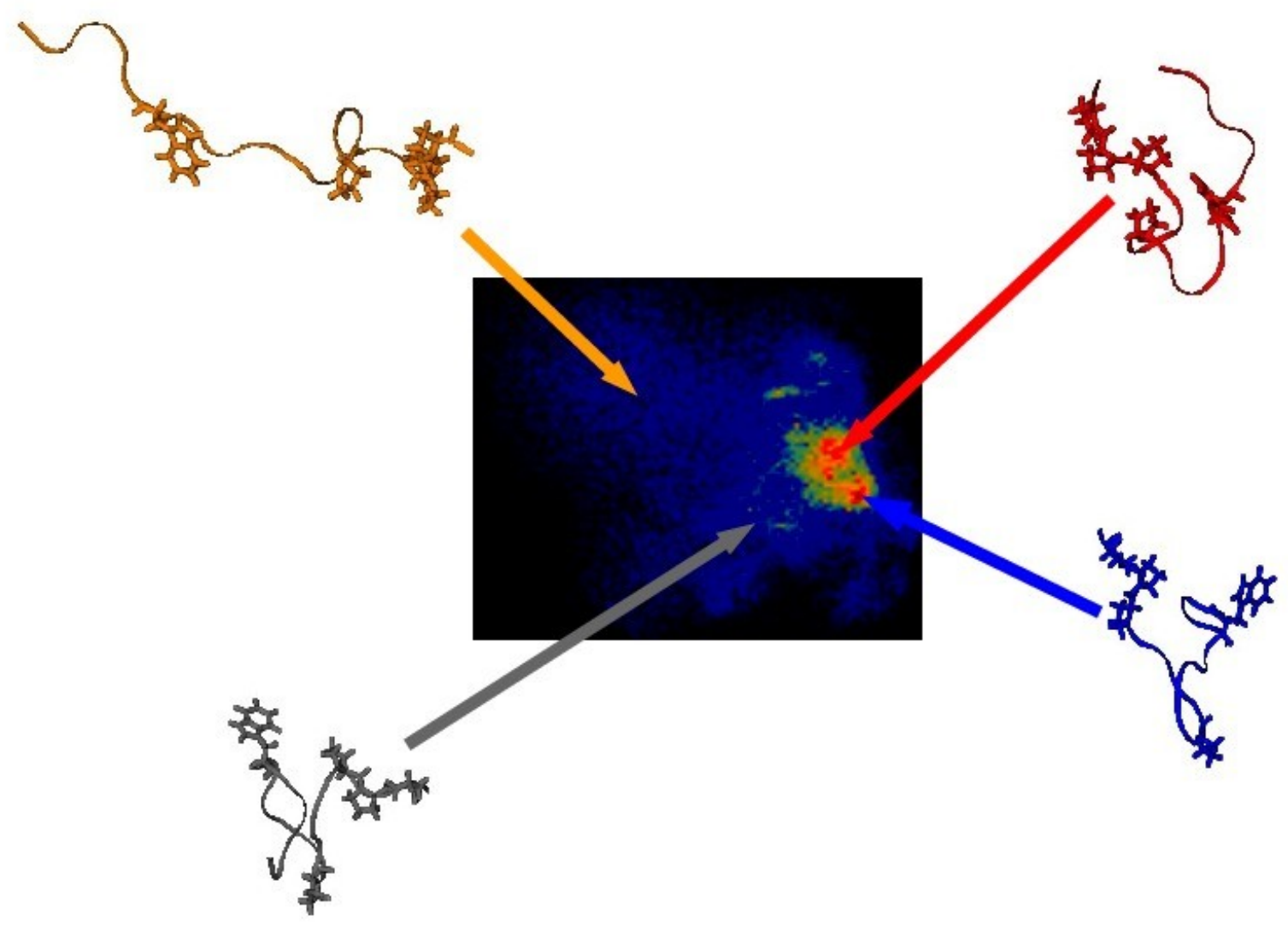

Figure S4. Some configurations explored in the simulation performed at $335 \mathrm{~K}$. Tryptophan and proline residues are plotted in stick representation. See text for further details. 


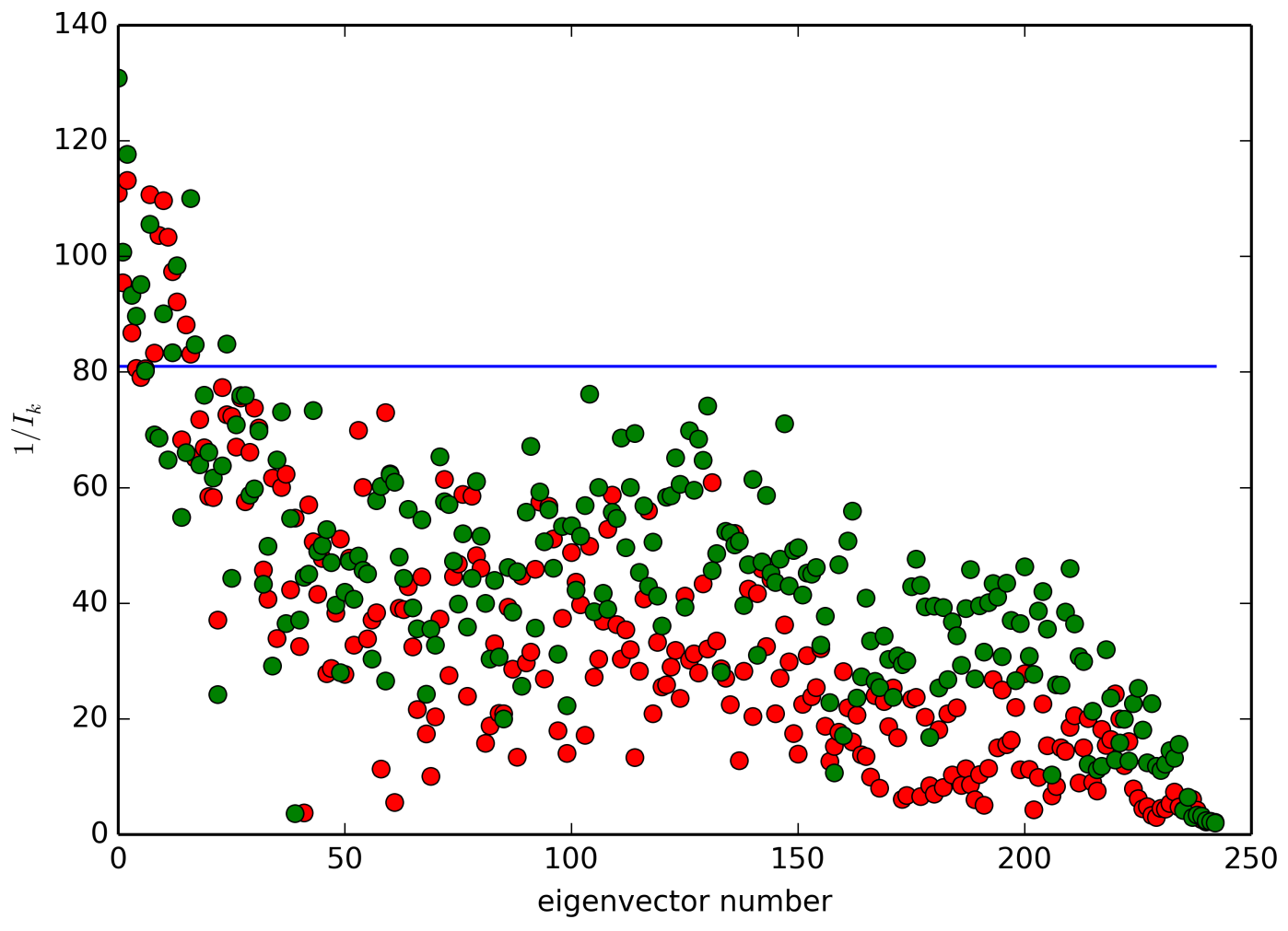

Figure S5. Participation index of eigenvectors for the $300 \mathrm{~K}$ simulation sampled at $10 \mathrm{ps}$ (red circles) and 200 ps (green circles). Blue line indicates to value to which eigenvectors from matrices belonging to the Wishart ensemble converge (the RMT expected value). 


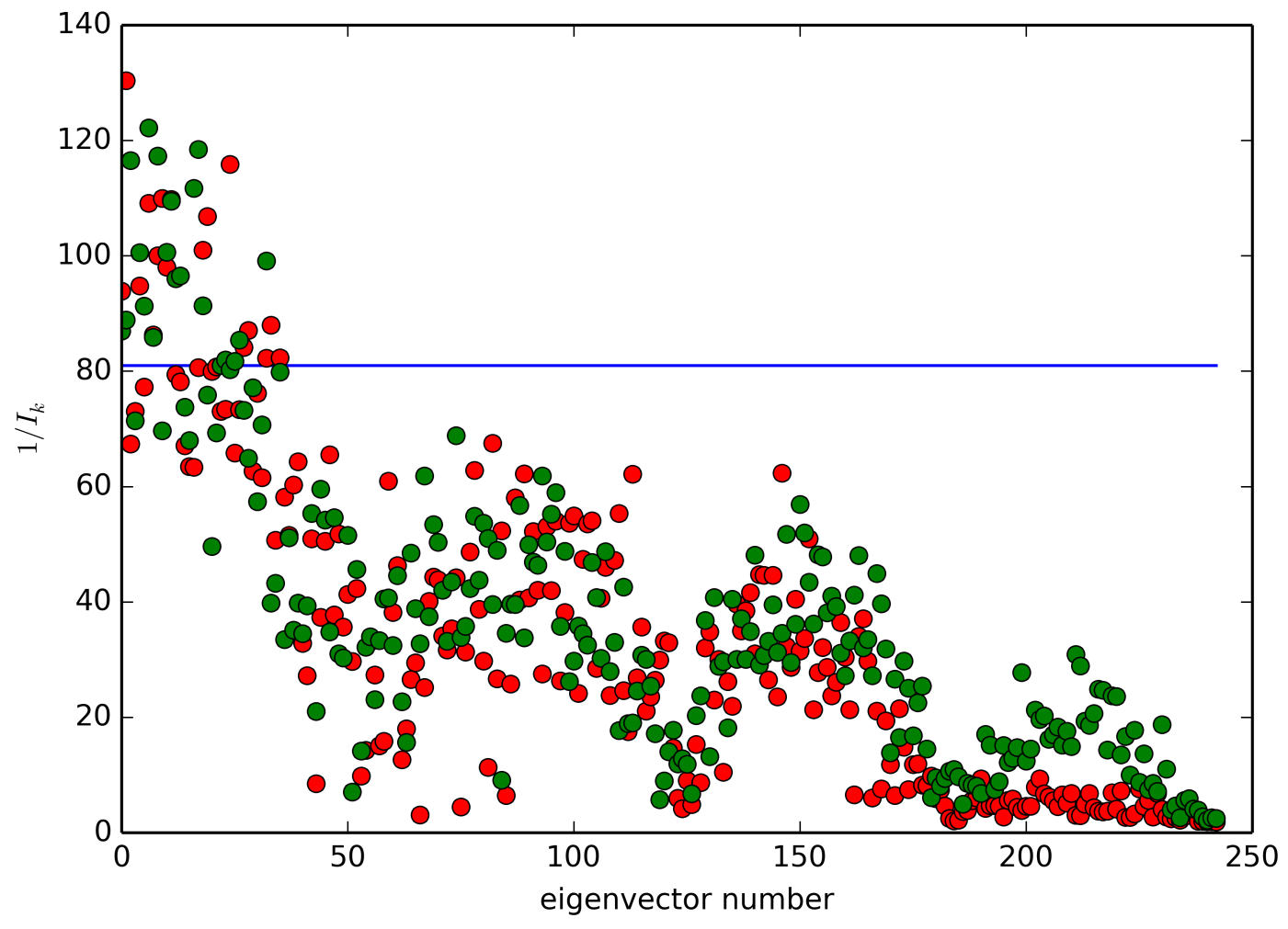

Figure S6. Participation index of eigenvectors for the $335 \mathrm{~K}$ simulation sampled at $10 \mathrm{ps}$ (red circles) and 200 ps (green circles). Blue line indicates to value to which eigenvectors from matrices belonging to the Wishart ensemble converge (the RMT expected value). 
Table S1. Determinant and trace of the covariance and correlation matrices. MD data are organized as time-difference matrices or as centroid normalized matrices.

\begin{tabular}{|c|c|c|c|c|c|c|c|c|c|}
\hline \multirow[t]{3}{*}{$\mathrm{T}(\mathrm{K})$} & $\Delta t$ (ns) & \multicolumn{4}{|c|}{ Correlation matrices } & \multicolumn{4}{|c|}{ Covariance matrices } \\
\hline & & \multicolumn{2}{|c|}{$x_{t+1}-x_{t}$} & \multicolumn{2}{|c|}{$x_{i}-\langle x\rangle$} & \multicolumn{2}{|c|}{$x_{t+1}-x_{t}$} & \multicolumn{2}{|c|}{$x_{i}-\langle x\rangle$} \\
\hline & & det & $\operatorname{tr}$ & det & $\operatorname{tr}$ & det & $\operatorname{tr}$ & det & tr \\
\hline \multirow[t]{5}{*}{300} & 10 & $1.6 \cdot 10^{-256}$ & 243.0 & 0 & 243.0 & 0 & 41.39 & 0 & 139.46 \\
\hline & 20 & $4.9 \cdot 10^{-269}$ & 243.0 & 0 & 243.0 & 0 & 52.70 & 0 & 139.46 \\
\hline & 50 & $1.1 \cdot 10^{-284}$ & 243.0 & 0 & 243.0 & 0 & 69.98 & 0 & 139.58 \\
\hline & 100 & $8.6 \cdot 10^{-298}$ & 243.0 & 0 & 243.0 & 0 & 84.09 & 0 & 139.63 \\
\hline & 200 & $3.5 \cdot 10^{-308}$ & 243.0 & 0 & 243.0 & 0 & 93.78 & 0 & 138.81 \\
\hline \multirow[t]{5}{*}{335} & 10 & $1.2 \cdot 10^{-314}$ & 243.0 & 0 & 243.0 & 0 & 199.80 & $4.9 \cdot 10^{-324}$ & 2788.74 \\
\hline & 20 & 0 & 243.0 & 0 & 243.0 & 0 & 297.91 & 0 & 2788.37 \\
\hline & 50 & 0 & 243.0 & 0 & 243.0 & $5.9 \cdot 10^{-320}$ & 500.38 & 0 & 2790.17 \\
\hline & 100 & 0 & 243.0 & 0 & 243.0 & $3.5 \cdot 10^{-308}$ & 731.54 & 0 & 2787.94 \\
\hline & 200 & 0 & 243.0 & 0 & 243.0 & $5.2 \cdot 10^{-300}$ & 1003.82 & 0 & 2791.90 \\
\hline
\end{tabular}

